\title{
Group for Research and Assessment of Psoriasis and Psoriatic Arthritis (GRAPPA)
}

\author{
P J Mease, D D Gladman, G G Krueger
}

Ann Rheum Dis 2005;64(Suppl II):ii1-ii2. doi: 10.1136/ard.2004.033894

$\mathrm{P}$ soriatic arthritis (PsA) is a condition characterised by inflammatory arthritis and enthesitis, which can occur in nearly a third of patients with psoriasis. These patients share unique genetic factors and immunopathogenic features in the joints, enthesium, and skin. The clinical characteristics of the joint disease associated with psoriasis have been more clearly elucidated over the past 30 years. Adding to this is an appreciation that standard systemic agents for psoriasis, such as methotrexate and ciclosporin, are also effective for PsA. However, it has been the recognition of the immunological features and the emergence of "biologicals" that target specific immune/inflammatory pathways of selected disease processes that have generated the current excitement and interest in autoimmune disease. These developments have given us new insights into pathogenesis while bringing new, more effective, and safer treatments for both the skin and joints. This supplement of the Annals of the Rheumatic Diseases is a compilation of articles summarising the state of the art in this field.

Several centres have contributed the majority of what we know about PsA due to longstanding research interest. Numerous inadequately explored questions remain about disease classification, disease pathophysiology, and the development of validated methodologies to assess disease outcomes, both in longitudinal cohorts and in clinical trials. There is an increasing international exchange of ideas among dermatologists and rheumatologists and between these two specialties about emerging findings in psoriasis and its related arthritis. This exchange has been broadened by advances in related fields in rheumatology, such as the other spondyloarthropathies and rheumatoid arthritis, and in dermatology, such as $\mathrm{T}$ cell mediated skin diseases. It has been supported by the biopharmaceutical industry, which sponsors both basic and applied research. Also participating in the exchange of ideas are representatives of regulatory agencies, which are concerned with the development, safety, and effectiveness of therapies, as well as patient advocacy organisations and specialty societies, which are concerned with raising public awareness, increasing access to treatments, and educating both the medical and lay populations.

One organisation, formed in 2003, the Group for Research and Assessment of Psoriasis and Psoriatic Arthritis (GRAPPA), is an example of this type of international collaboration. Many of the articles in this supplement are based on presentations and discussions which took place at the inaugural meeting of this group in August 2003 in New York City as well as at subsequent meetings, including discussions held in a psoriatic workshop at the seventh meeting of Outcome Measures in Rheumatology (OMERACT) in May 2004. GRAPPA now numbers over 125 physicians and other individuals who have a dedicated interest in clinical care, education, and research involving patients with psoriasis and PsA.
The mission of GRAPPA includes the following elements:

- increasing awareness and early diagnosis of psoriasis and PsA

- development and validation of research assessment tools to measure clinical status and disease outcome

- evaluation of treatment modalities

- supporting and conducting basic research on disease pathophysiology

- fostering communication between rheumatologists, dermatologists, representatives of patient advocacy organisations, biopharmaceutical companies, regulatory agencies, and others who are interested in the advancement of care of psoriasis and PsA.

GRAPPA was modelled after the ASessment in Ankylosing Spondylitis (ASAS) working group, first formed in 1995, which has been involved in research in the field of ankylosing spondylitis and related spondyloarthropathies. Details about this group's origins and accomplishments are reviewed in the article in this supplement by van der Heijde et al. ${ }^{1}$ When GRAPPA was first conceived, numerous independent threads of related activity were underway. A number of clinical researchers, particularly in Europe and North America, had established patient registries to gather data and publish observations on the natural history of psoriasis and PsA, as well as to conduct research on the genetics and pathophysiology of these diseases. Patient advocacy groups, focusing on psoriasis as well as PsA, had sprung up in numerous countries to foster the education of patients and promote awareness in the general public. Clinical investigators in the fields of rheumatology and dermatology, utilising drugs developed by biopharmaceutical companies to treat conditions resulting from autoimmune inflammation, were increasingly focused on psoriasis and PsA and achieving significant inroads towards disease improvement. The ClASsification of Psoriatic Arthritis (CASPAR) study group initiated by Philip Helliwell of Leeds, England, and 32 PsA rheumatology researchers, focused on the development of updated classification criteria for PsA; this core of interested rheumatologists were more recently joined by similarly interested dermatologists.

The goals of the GRAPPA initiative are as follows:

(1) Provide a forum for acquaintance, networking, and communication between international researchers in rheumatology and dermatology, industry, patient advocacy organisations, and regulatory agencies.

(2) Develop and conduct collaborative research, education and other projects, and provide the opportunity for inperson meetings and intranet communication to share knowledge and research findings with others. 
(3) Develop and validate a criteria set for the definition of PsA.

(4) Prioritise domains of enquiry within PsA and psoriasis for research.

(5) Review, develop, and validate effective and feasible outcome measures for the assessment of PsA and psoriasis.

(6) Promote the development of national and international collaborative registries of PsA and psoriasis patients to standardise the data being obtained and learn more about the natural history of the disease as well as its genetic underpinnings.

(7) Work closely with representatives of patient advocacy organisations to promote public education and awareness of PsA and psoriasis and improve our understanding of patient needs.

(8) Work closely with representatives of biopharmaceutical companies to promote and conduct research on effective therapies for PsA and psoriasis.

(9) Work closely with representatives of regulatory agencies to establish appropriate guidelines for regulatory approval of new therapies.

(10) Work with other professional bodies, such as the American College of Rheumatology, American Academy of Dermatology, OMERACT, etc. to promote knowledge of and research about PsA and psoriasis within the context of those disciplines.

(11) Develop treatment guidelines for governmental and other interested parties.

This supplement covers many of these areas of focus and other aspects of the PsA and psoriasis research and education agenda. It is organised into sections on disease classification, genetics, pathophysiology, clinical features and immunology, assessment, therapy, clinical registries and genomics, and development of assessment methodologies with paired articles written by rheumatologists and dermatologists focusing on the joints and skin, respectively. In many areas, there is overlap in the discussion, such as when discussing domains such as quality of life. Additional featured discussions include a descriptive article on patient advocacy organisations and their role in advancing patient care, ${ }^{2}$ an article focusing on when it is best to "lump" PsA together with other spondyloarthropathies and when best to "split" it from them as a separate condition, ${ }^{3}$ and a review of psoriatic arthritis in the context of the OMERACT process. ${ }^{4}$ There are also four articles in question and answer format, based partly on discussions that took place during the meeting in August 2003 in New York and partly on interchange that occurred on the group's intranet. These articles highlight subjects that have not yet been completely researched and elucidated but are of keen interest.

Recognising that the findings in this field are advancing rapidly, these articles are available online as well as in print to facilitate broad exchange of information, and we expect to provide updates periodically in future publications. Further, the online version is freely available to all at no fee, courtesy of GRAPPA. Please help us to publicise its availability.

\section{ACKNOWLEDGEMENTS}

We would like to express our deep appreciation to the members of GRAPPA and its steering committee for stimulating work and dialogue, both in meetings and over the internet; to the following companies-Abbott, Amgen, Aventis, Biogen, Centocor, Genentech, Novartis, Schering-Plough, Serono, and Wyeth-which have provided unrestricted financial support for meetings, infrastructure, and publication; to Robin Shapiro and Health Advocacy Strategies in Seattle for administrative support; and to Diana Benzaia in New York for editorial support.

Members of the GRAPPA steering committee

Christian Antoni, Erlangen, Germany

Jonathan Barker, London, UK

Jürgen Braun, Herne, Germany

Enno Christophers, Kiel, Germany

Dafna D Gladman, Toronto, Ontario, Canada

Alice Gottlieb, New Brunswick, NJ, USA

Philip S Helliwell, Leeds, UK

Joachim Kalden, Erlangen, Germany

Arthur Kavanaugh, La Jolla, CA, USA

Gerald G Kreuger, Salt Lake City, UT, USA

Philip J Mease, Seattle, WA, USA

Alan Menter, Dallas, TX, USA

Peter Nash, Queensland, Australia

Jean-Paul Ortonne, Nice, France

Christopher Ritchlin, Rochester, NY, USA

J H Saurat, Zurich, Switzerland

Josef Smolen, Vienna, Austria

Vibeke Strand, Portola Valley, CA, USA

William J Taylor, Wellington, New Zealand

Peter van de Kerkhof, Nijmegen, the Netherlands

\section{Authors' affiliations}

P J Mease, Seattle Rheumatology Associates and Rheumatology Clinical Research, Swedish Hospital Medical Center, University of Washington School of Medicine, Seattle, WA, USA

D D Gladman, Toronto Western Research Institute, Psoriatic Arthritis Program, University Health Network, Centre for Prognosis Studies in the Rheumatic Diseases, Toronto Western Hospital, Toronto, Ontario, Canada

G G Krueger, Department of Dermatology, University of Utah Health Sciences Center, Salt Lake City, UT, USA

Correspondence to: Dr P Mease, Seattle Rheumatology Associates, 1101 Madison, 10th floor, Seattle WA 98104, USA; pmease@nwlink. com

\section{REFERENCES}

1 van der Heijde D, Braun J, Landewé R, Davis J, Sieper J, van der Linden S et al. ASsessment in Ankylosing Spondylitis (ASAS) international working group: a model for psoriatic arthritis and psoriasis? Ann Rheum Dis 2005;64(suppl II):ii 108-9.

2 Zimmerman GM, Savage LM, Chandler DC, Maccarone Buonfigli M. Psoriatic arthritis and psoriasis: the role of patient advocacy organisations in the twenty first century. Ann Rheum Dis 2005;64(suppl II):ii93-100.

3 Nash P, Mease PJ, Braun J, van der Heijde D. Seronegative spondyloarthropathies: to lump or split? Ann Rheum Dis 2005;64(suppl II):ii9-13.

4 Gladman DD, Strand V, Mease PJ, Antoni C, Nash P, Kavanaugh A. OMERACT 7 psoriatic arthritis workshop: synopsis. Ann Rheum Dis 2005;64(suppl II):ii 1 15-6. 